\title{
CONTRIBUCIÓN AL CONOCIMIENTO DE LOS NEURÓPTEROS DE ALICANTE
}

\author{
(NEUR., PLANIPENNIA)
}

\author{
por \\ V. J. Monserrat ${ }^{1}$
}

\section{INTRODUCCION}

Continuando en la línea de trabajo mediante la cual trato de ir dando a conocer la fauna neuropterológica de España, se inicia el presente trabajo con el estudio de los neurópteros de la provincia de Alicante.

La provincia de Alicante, con una extensión de $5.862 \mathrm{~km}^{2}$ presenta un gran interés faunístico tanto por su situación geográfica, por su orografia y especialmente por tratarse de una provincia mucho menos degradada y alterada por acción antropógena que las otras provincias circundantes, permitiendo que en las zonas del interior, conserve amplias zonas con biotopos poco modificado o al menos no tan lamentablemente alterados como viene siendo general en nuestra zona mediterránea.

Circunscribiéndonos al campo de los neurópteros, puede servir como introducción al interés que presenta el estudio faunístico de esta provincia, el hecho de que algunas especies como Josandreva sazi Navas, 1906 ó Wesmaelius navasi (Andreu, 1911) tienen a Orihuela como localidad típica. Otras como Chrysoperla mediterranea (Hözel, 1972), Cunctochrysa baetica (Hözel, 1972) o Aleuropteryx remane (Rausch, Aspöck y Ohm, 1978), han sido recientemente descritas contando con material colectado en esta provincia y algunas de las últimas novedades para Europa como Pterocroce capillaris (Klug, 1836) se han hallado en ella.

A pesar de este interés, no existe ningún trabajo previo que haya contemplado el estudio de este orden de insectos en la provincia de Alicante y prácticamente todos los datos que poseemos sobre su fauna

(1) Departamento de Zoologia, Facultad de Ciencias, Universidad de Murcia.

Para la realización de este trabajo se ha contado con la colaboración de la Comisión Asesora Científica y Técnica. 
neuropterológica se circunscriben, al margen de las anteriormente citadas, a los datos de Orihuela de ANDREU, 1909 y 1911 ó a los de VIDAL Y LOPEZ, 1943 que se repiten frecuentemente o que se añaden a otros que pueden recabarse en los artículos que se indican en la bibliografía.

No obstante, la mayoría de las especies anteriormente citadas en esta provincia representan las más frecuentes o conspicuas y en ocasioness su correcta determinación me sugiere algunas reservas, no obstante estas citas quedan indicadas a lo largo del texto.

Anotado el estado de conocimiento que en esta parcela poseemos sobre la fauna de Alicante, sólo me queda indicar que se trata de una provincia realmente accidentada donde sucesivas sierras se distribuyen especialmente en la zona central y boreal de la misma. De sus $5.862 \mathrm{~km}^{2}$ parte conserva una vegetación de fanerófitos y nanofanerófitos que representan en parte su vegetación potencial típicamente mediterránea. Sabinas, enebros, lentiscos, tamarindos, coscojas, encinas y adelfas son relativamente frecuentes en zonas donde la incidencia humana no ha degradado en exceso estos medios, habiendo sido sustituída en ocasiones por otras especies de interés económico o arrasada por crecimiento urbano y turístico.

\section{METODO}

Para la toma de datos, se han elegido 30 localidades de muestreo que pudieran resultar representativas de los diferentes ecosistemas existentes en la provincia, tratando de evitar aquellas en las que la acción antropógena no nos reportara más datos que los correspondientes a las especies acompañantes a estas acciones y poder dedicar una mayor atención a medios más espontáneos que albergaran una fauna más autóctona. No obstante esta idea no ha sido siempre factible, ya que no puede indicarse que ninguna de las localidades visitadas, no estuviera alterada por la acción humana en mayor o menor medida.

En estas localidades se ha muestreado al menos dos veces en primavera y dos en verano, tratando de colectar el mayor número de ejemplares, anotando de cada uno de ellos la localidad, fecha y planta sobre la que se hallaba con el fin de aportar nuevos datos sobre su biología.

Se han obtenido ejemplares en 25 localidades de entre las 30 elegidas (mapa n. 1), cuyas coordenadas U.T.M. y altitud aproximada se indican a continuación: 
Se han obtenido ejemplares en 25 localidades de entre las 30 elegidas (mapa n. 1), cuyas coordenadas U.T.M. y altitud aproximada se indican a continuación:

1 Algorfa (30SXH9317, 25 m.; 2 Ameradores (30SYH1672), 700 m.; 3 Barranco de Guadalest (30SYH4783), $350 \mathrm{~m}$.; 4 Benidorm (30SYH4467), 10 m.; 5 Calpe (31SBC4181), 40 m.; 6 Chinorlet (30SXH7754), 620 m.; 7 Cocentaina (30SYH2490), 450 m.; 8 Crevillente (30SXH9134), $130 \mathrm{~m} . ; 9 \mathrm{La}$ Aparecida (30SXH7416), 80 m.; 10 Lomas de Gigi (30SYH3462), 200 m.; 11 Margarida (30SYH3496), 630 m.; 12 Monóvar (30SXH8457), 500 m.; 13 Montesol (30SYH1205), 800 m.; 14 Parcent (30SYH5592), 290 m.; 15 Pego (30SYJ5102), 85 m.; 16 Pilar dé la Horadada (30SXG9491), 15 m.; 17 Puerto de Biar (30SXH9477), 810 m.; 18 Puerto de Confrides (30SYH3785), 960 m.; 19 Puerto de la Carrasqueta (30SYH7814), 1000 m.; 20 Salt (30SYH1685), 560 m.; 21 Sax (30SXH9067), 470 m.; 22 Tárbena (30SYH5287), 560 m.; 23 Teulada (31SBC4691), 180 m.; 24 Torrellano (30SYH1341), 50 m.; 25 Venta de Pedreguer (31SBC4398), 75 m.

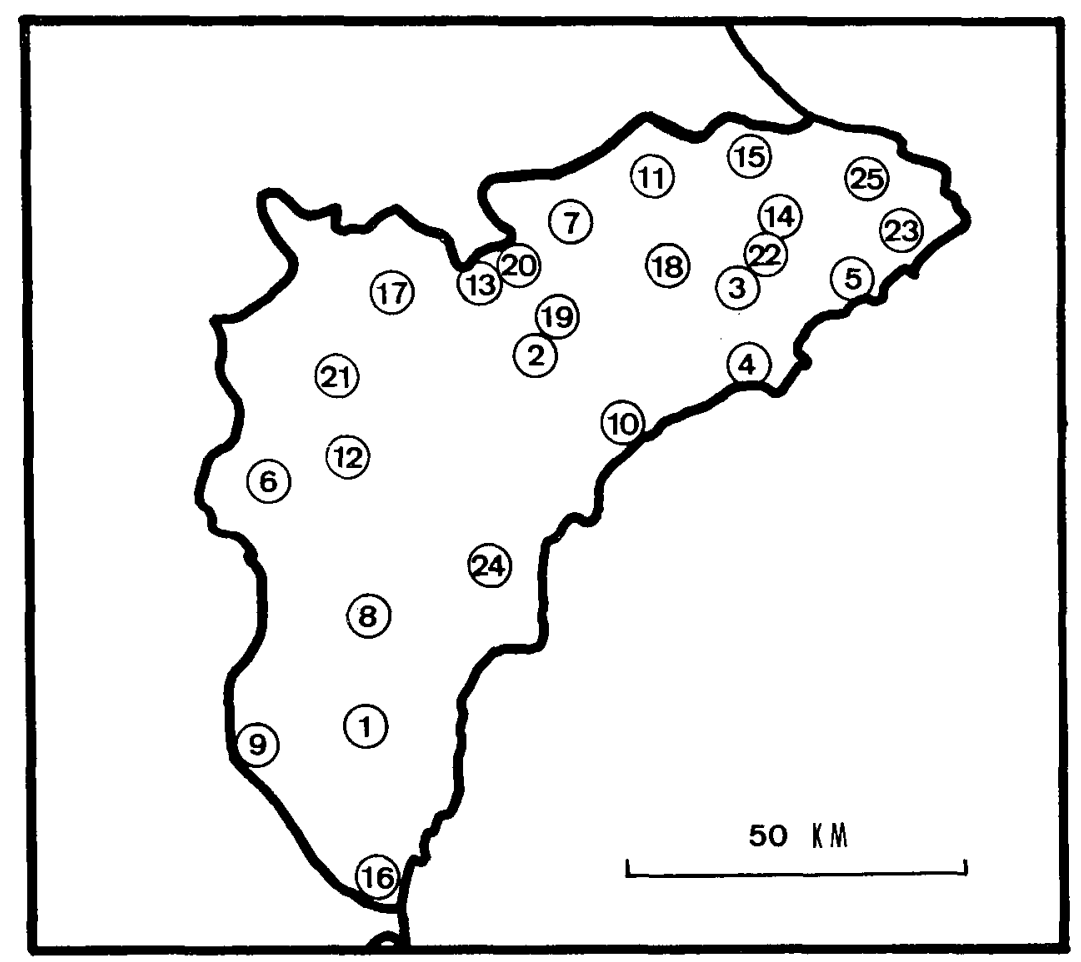


Han sido colectados 844 ejemplares, pertenecientes a 47 especies diferentes, incluídas en 7 familias según se indica en el siguiente cuadro:

\begin{tabular}{|c|ccccccc|c|}
\hline & MYrM. & ASCAL. & NEMOPT. & CRIS. & HEMER. & MANT. & CONIO. & Total \\
\hline N. esp. & 6 & 1 & 2 & 14 & 5 & 2 & 17 & 47 \\
\hline № eje. & 167 & 2 & 12 & 264 & 12 & 8 & 379 & $\mathbf{8 4 4}$ \\
\hline
\end{tabular}

Puede destacarse a la familia Coniopterygidae como la más abundante tanto en número de especies como en ejemplares colectados.

De estas 47 especies, 19 son nuevas para la fauna de la provincia de Alicante y quedan indicadas en el texto mediante un asterisco.

\section{MATERIAL ESTUDIADO:}

NEMOPTERIDAE Burmeister, 1839

Nemoptera bipennis (Illiger, 1812)

Especie endémica de la Península Ibérica, había sido anteriormente citada en la provincia por ANDREU, 1909 y VIDAL Y LOPEZ, 1943. Unicamente se han colectado dos machos en el Puerto Biar 18-V-1982.

Pterocroce capillaris (Klug, 1836)

Especie erémica conocida de Arabia, Irán, Egipto, Chad y Argelia y que ha sido recientemente hallada en el S.E. español.

Algorfa 4-VIII-1982 2 ○’ $0^{\prime \prime}, 2$ 우, Crevillente 17-VII-1982 1 ơ, 1 ㅇ, 4-VIII-1982 2 ơ ơ, 2 우.

Habita en cuevas y oquedades y como se indicó en MONSERRAT, 1983 a; larvas pertenecientes a esta especie fueron encontradas en Benimantell, Crevillente y Pilar de la Horadada. 
ASCALAPHIDAE Rambur, 1842

Bubopsis agrioides (Rambur, 1842)

Especie de distribución atlantomediterránea y conocida en España, Francia y Marruecos, había sido citada en Orihuela y en Cox por ANDREU, 1909, NAVAS, 1913 b y VIDAL Y LOPEZ, 1943.

Ameradores 27-VI-19821 q, Monóvar 28-VI-19821 .

Es característica de eriales y lomas soleadas y secas, no siendo fáciles su localización y captura.

MYRMELEONIDAE Latreille, 1803

Myrmeleon (Morter) hyalinus Olivier, 1811

Especie holomediterránea extendida hasta Arabia e Irán. Citas anteriores en la provincia pueden extraerse de ANDREU, 1909, VIDAL Y LOPEZ, 1943 y ASPÖCK, ASPÖCK y HÖLZEL, 1980 bien bajo esta denominación o como Myrmeleon distinguendus Rambur, 1842.

Benidorm 17-VII-1982 1 ○, 1 q, Pilar de la Horadada 11-VII-1982 2 ○" ○", 1 ㅇ.

Es características de zonas costeras o arenosas del interior.

Macronemurus appendiculatus (Latreille, 1807)

Especie característica del Mediterráneo occidental había sido citada de Orihuela por ANDREU, 1909 y postriormente listada por VIDAL Y LOPEZ en 1943. Ha sido colectada en numerosas localidades.

Algorfa 11-VII-1982 1 ơ, 3 \% , 4-VIII-1982 1 ơ, 2 \%, Ameradores 27-VI-1982 4 ơ ơ, 4-VIII-1982 4 ơ ơ, 5 ᄋ Guadalest 18-VII-1982 2 ơ ơ, 1 q, 4-VIII-1982 2 ơ ơ, 2 ๆ , Beni-

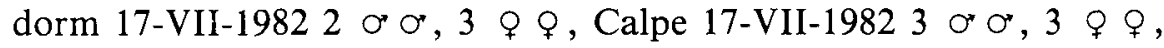
Chinorlet 28-VI-1982 1 ơ, Cocentaina 27-6-1982 1 ○ , 18-VII-1982 2

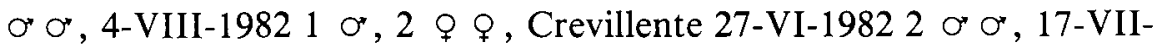
19827 ○ 
19823 ơ ơ, 5 \& \&, Margarida 5-VIII-1982 2 ơ on, 3 \& , Monóvar 5VIII-1982 1 on, 2 ㅇ ㅇ, Parcent 17-VII-1982 1 o", 5-VIII-1982 2 o $\sigma^{\prime}, 3$

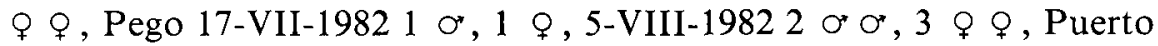
de Biar 5-VIII-1982 1 ○, 1 \&, Pilar de la Horadada 16-VI-1982 1 ○, 11-VII-1982 1 ơ, 5 ○ \&, 4-VIII-1982 2 ơ ơ, 2 q $\dot{q}$, Puerto de Confrides 18-VII-1982 1 ○, 4-VIII-1982 6 ơ ơ, 5 \& $\$$, Puerto de la Carrasqueta 18-VII-1982 1 o , 1 \%, 4-VIII-1982 2 o o $^{\prime} 2$ \% , Salt 5-VIII19822 ơ ơ 4 ㅇ, Sax 5-VIII-1982 3 ơ ơ, 2 \& , Tárbena 5-VIII$19821 \sigma^{*}, 2$ $\%$, Teulada 17-VI-1982 1 o*, 1 \%, Torrellano 27-VI19823 ơ ơ, 1 ९, 17-VII-1982 2 ९ , 4-VIII-1982 3 or or, 9 ९.

Se trata de una especie ubiquista aunque prefiere las zonas secas.

Neuroleon arenarius (Navas, 1904)

Especie conocida del Mediterráneo occidental de la que ha sido colectado un único ejemplar. Se trata de un macho capturado en Venta de Pedreguer 17-VII-1982.

Otras localidades de esta especie en la provincia pueden hallarse en ANDREU, 1909 y VIDAL Y LOPEZ, 1943.

Neuroleon egenus (Navas, 1915)

Especie holomediterránea citada anteriormente en la provincia como Myrmeleon sticticus Navas, 1903 por NAVAS, 1907, ANDREU, 1909, NAVAS, 1913 a, 1924 y VIDAL Y LOPEZ, 1943.

Tárbena 17-VII-1982 1 ơ, Venta de Pedreguer 17-VII-1982 1 ○”.

La suposición hecha por MONSERRAT, $1983 \mathrm{c}$ de que podría tratarse de una especie con tendencia arborícola, parece confirmarse, ya que de los ejemplares citados uno ha sido colectado sobre Quercus ilex y otros sobre Ceratonia siliqua.

Nemoleon notatus (Rambur, 1842)

Rara especie conocida del sur de la Península Ibérica, Marruecos, Argelia, Etiopía, Chad, Uganda y Angola. 
Pilar de la Horadada 16-VI-1982 1 ơ, 11-VII-1982 1 ơ.

Esta especie está citada por ANDREU, 1909 en esta localidad y en Rojales, VIDAL Y LOPEZ, 1943 repite estas citas y NAVAS, 1924 y ASPÖCK, SPÖCK y HÖLZEL, 1980 la citan de Alicante. Nada se conoce sobre esta especie respecto a su biología y estados larvarios.

Creoleon lugdunensis (Villers, 1789)

Especie conocida del Mediterráneo occidental citada en la provincia anteriormente por MONSERRAT, 1979. La cita de Creagris plumbeus (Olivier, 1811) dada por ANDREU, 1909 en Orihuela, debe considerarse como perteneciente a la especie que nos ocupa, ya que ésta es exclusiva del Mediterráneo oriental.

Curiosamente se han colectado pocos ejemplares de esta especie, a pesar de tratarse de una especie normalmente frecuente: Pilar de la Horadada 11-VII-1982 $1{ }^{\circ}, 1 \%$, Salt 28-VI-1982 $1 \sigma^{*}$.

Se trata de una especie característica de zonas secas que ocupa los mismos medios que Macronemurus appendiculatus si bien más tempranamente, siendo sustituída por aquélla.

HEMEROBIIDAE Latreille, 1803.

Sympherobius (Sympherobius) fallax Navas, 1908

Especie holomeditarránea extendida a Etiopía y Sudán. Está citada como Nefasitus tenellus Navas, 1908 por NAVAS, 1924 en Alicante y como Sympherobius tenellus por NAVAS, 1908 y ANDREU, 1911 en esta localidad. Presumo que la cita de VIDAL Y LOPEZ, 1943 en Alicante de Nephasitus fallans Nav. debe referirse a la especie que tratamos.

Torrellano 17-VII-1982 1 ơ, sobre Ceratonia siliqua.

Sympherobius (Shympherobius) elegans (Stephens, 1836)

Especie distribuída por todo el continente europeo, había sido citada de Rojales y Orihuela por ANDREU, 1911 y VIDAL Y LOPEZ, 1943. El tipo de $S$. venustus es de Orihuela y esta especie descrita por NAVAS, 1908 es sinónima de la que tratamos. 
Pego 27-VI-1982 1 o*, Torrellano 27-VI-1982 1 q, 17-VII-1982 1 ơ, sobre Ceratonia siliqua.

Sympherobius (Sympherobius) pygmaeus (Rambur, 1842)

Especie holomediterránea extendida hacia el ceniro y norte de Europa. Había sido citada en la provincia bajo esta denominación o como $S$. venosus Navas, 1908 por NAVAS, 1908, 1924, ANDREU, 1911, y VIDAL Y LOPEZ, 1943.

Teulada 17-VII-1982 1 ơ, 1 \&, sobre Ceratonia siliqua.

* Hemerobius stigma (Stephens, 1836)

Especie holártica, asociada en la zona de estudio al Pinus halepensis sobre el que se han colectado todos los ejemplares.

Barranco de Guadalest 27-VI-1982 1 \%, Pilar de la Horadada 16VI-1982 2 ơ ơ.

Wesmaelius (Kimminsia) subnebulosus (Stephens, 1836)

Especie holártica, citada anteriormente de Orihuela por NAVAS, 1908, ANDREU, 1911, y VIDAL Y LOPEZ, 1943.

Crevillente 27-VI-1982 1 ơ, La Aparecida 18-V-1982 1 o , Pilar de la Horadada 16-VI-1982 1 \&. De estos ejemplares dos se han colectado sobre Pinus halepensis y uno sobre Ceratonia siliqua.

MANTISPIDAE Leach, 1815

* Mantispa styriaca (Poda, 1761)

Especie holomediterránea expansiva a centro-Europa parece ser relativamente frecuente en la provincia.

Benidorm 17-VII-1982 1 ơ, Cocentaina 18-VII-1982 1 \%, 4-VIII19821 \&, Teulada 17-VII-1982 1 @. Dos ejemplares fueron colectados sobre Quercus ilex y dos sobre Pinus halepensis. 
Especie holomediteránea y como se indicó en MONSERRAT, 1983 confundida con Mantispa mandarina Navas, 1914 por ASPÖCK, ASPÖCK y HÖLZEL, 1980. Está citada como Perlamantispa perla (Pallas, 1772) por MONSERRAT, 1979 en Benifato por considerarse entonces como una variedad de $P$. perla según fue originalmente descrita y no de una auténtica especie como en la actualidad he constatado.

Cocentaina 18-VII-1982 $2 q q$, Puerto de Biar 5-VIII-1982 1 으, Puerto de la Carrasqueta 18-VII-1982 1 ơ. Tres ejemplares se colectaron sobre Quercus ilex y uno sobre Pinus halepensis.

CHR YSOPIDAE Schneider, 1851

Italochrysa italica (Rossi, 1790)

Especie de distribución holomediterránea, había sido citada en la provincia por ANDREU, 1909, NAVAS, 1924 y VIDAL Y LOPEZ, 1943.

Parcent 17-VII-1982 1 ○, Torrellano 17-VII-1982 1 ○, 2 ९, todo sobre Ceratonia siliqua. Probablemente dos huevos eclosionados que fueron hallados sobre gramíneas en el Barranco de Guadalest 18-VII1982 podría pertenecer a esta especie, según puede desprenderse de los datos aportados por PRINCIPI, 1946.

Chrysopa septempunctata Wesmael, 1841

Especie de dispersión paleártica y que había sido citada en Alicante por ANDREU, 1909, NAVAS, 1908, 1915 a, VIDAL Y LOPEZ, 1943 y HÖLZEL y OHM, 1972.

Crevillente 18-V-1982 1 ○, Parcent 17-VII-1982 1 ○, Pilar de la Horadada 11-VII-1982 1 \&, Puerto de la Carrasqueta 18-VII-1982 1 , Teulada 17-VII-1982 1 ơ. De estos ejemplares tres se colectaron sobre Ceratonia siliqua, uno sobre Quercus ilex y uno sobre Pinus halepensis. 
* Chrysopa viridana Schneider, 1845

Especie holomediterránea expansiva, alcanzando Irán y Afganistán. Se han colectado pocos ejemplares: Pego 5-VIII-1982 1 ơ, 2 \& sobre Ceratonia siliqua, Tárbena 5-VIII-1982 1 ơ sobre Quercus ilex.

Chrysopa formosa Brauer, 1850

Distribuída sobre las zonas meridionales de la región paleártica, existen numerosas referencias para la provincia: ANDREU, 1909, NAVAS, 1915 a, 1924 y VIDAL Y LOPEZ, 1943.

Unicamente se ha colectado una hembra en Pilar de la Horadada 16-VI-1982 sobre Pinus halepensis.

Chysoperla carnea (Stephens, 1836)

Especie ampliamente distribuída por Eurasia, Africa y Norteamérica que puede considerarse cosmopolita por acción humana. Fue citada como Chrysopa vulgaris Schneider, 1851 en esta provincia por NAVAS, 1908, ANDREU, 1909 y VIDAL Y LOPEZ, 1943.

Algorfa 16-VI-1982 1 ○’, 2 ๆ, Barranco de Guadalest 18-VII19822 ○ \&, Chinorlet 28-VI-1982 3 ơ ơ, Cocentaina 27-VI-1982 1 ơ, 1 , 18 -VII-1982 2 우, La Aparecida 27-VI-1982 1 ๆ, Lomas de Gigi 17-VII-1982 2 ơ ơ, Monóvar 28-VI-1982 1 ○ , 4 ๆ , Montesol 28-VI19823 o ơ, Parcent 17-VII-1982 1 \%, Pego 27-VI-1982 1 ơ, 1 \%, 17VII-1982 1 \&, Pilar de la Horadada 16-VI-1982 1 \&, 11-VII-1982 1 o , 1 ९, Puerto de Confrides 4-VIII-1982 $1 \sigma^{\circ}$, Puerto de la Carrasqueta 27-

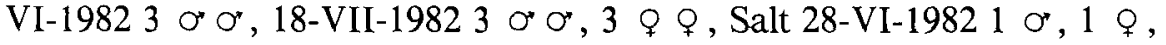
Sax 28-VI-1982 2 ơ ơ 4 \& ㅇ, Tárbena 27-VI-1982 1 , 17-VII-1982 3

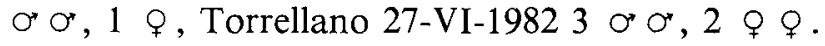

Especie muy poco exigente y como puede apreciarse frecuente en la zona estudiada. Han sido colectados 34 ej. sobre Pinus halepensis, 16 sobre Quercus ilex, 7 sobre Ceratonia siliqua y 1 sobre Juniperus oxycedrus. 
Especie atlantomediterránea, citada anteriormente por HÖZEL, 1972 a, HÖLZEL y OHM, 1972 y ASPÖCK, ASPÖCK y HÖLZEL, 1980 en la provincia

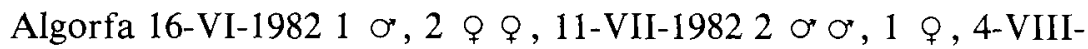
19822 ơ $^{*}$, La Aparecida 18-V-1982 1 \%, 27-VI-1982 3 o $\sigma^{\circ}, 1$ ๆ, Lomas de Gigi 17-VII-1982 3 ơ ơ, Parcent 27-VI-1982 1 \&, 17-VII-1982 3 ㅇ $९$, Pilar de la Horadada 11-VII-1982 2 우, Torrellano 17-VII-1982

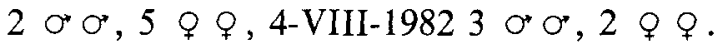

Como se indica en otras ocasiones, esta especie parece circunscrita a Pinus halepensis y al menos en la zona de estudio así ha quedado demostrado, ya que todas las capturas se han realizado sobre esta conífera.

Anisochrysa prasina (Burmeister, 1839)

Especie de distribución paleártica que había sido anteriormente citada por NAVAS, 1908 ANDREU, 1909, VIDAL Y LOPEZ, 1943 y HÖLZEL y OHM, 1972.

Barranco de Guadalest 18-VII-1982 1 ९, Cocentaina 27-VI-1982 1 ९, 18-VII-1982 2 ९ , Margarida 27-VI-1982 1 ९, Montesol 28-VI$19821 \sigma^{\circ}$, Puerto de Confrides 4-VIII-1982 1 \&, Puerto de la Carrasqueta 18-VII-1982 2 ơ ơ, 2 우, Tárbena 17-VII-1982 1 o, 1 9.

Esta especie ocupa medios generalmente húmedos habiendo sido colectada sobre Quercus ilex en 12 ocasiones y sobre Cerantonia siliqua en una.

Anisochrysa venosa (Rambur, 1842)

Especie distribuda por el sur de la región paleártica, desde Marruecos a Mongolia que ha sido citada en la provincia de Alicante frecuentemente: NAVAS, 1908, ANDREU, 1909, NAVAS, 1915 a, VIDAL Y LOPEZ, 1943 y HÖLZEL y OHM, 1972.

La Aparecida 27-VI-1982 1 ơ, Parcent 27-VI-1982 1 ơ, Tárbena 27-VI-1982 1 \&, Torrellano 17-VII-1982 1 o*, 1 ․ 
Es una especie de tendencia xerófila y biología prácticamente desconocida. Se han colectado tres ejemplares sobre Pinus halepensis, uno sobre Ficus carica y uno sobre Ceratonia siliqua.

Anisochrysa genei (Rambur, 1842)

Especie holomediterránea de la que pueden anotarse referencias para la provincia en ANDREU, 1909, NAVAS, 1915 a, 1924, VIDAL Y LOPEZ, 1943 y HÖLZEL y OHM, 1972.

Algorfa 4-VIII-1982 1 ๙ , Lomas de Gigi 17-VII-1982 1 ๙ , Parcent 17-VII-1982 1 q, Pilar de la Horadada 11-VII-1982 5 ơ ơ, 4 ๆ ,

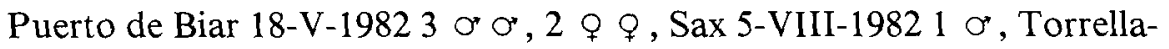
no 27-VI-1982 1 ㅇ, 17-VII-1982 2 웅, 4 우요.

Parece estar muy repartida y como es usual en esta especie está asociada a zonas secas. Han sido capturados 16 ejemplares sobre Pinus halepensis, 4 sobre Quercus coccifera, 3 sobre Ceratonia siliqua y 1 sobre Pistacia lenticus.

Anisochrysa flavifrons (Brauer, 1850)

Especie holomediterránea, extendida al centro y norte de Europa. Bien bajo esta denominación o como Chrysopa lineolata McLachlan, 1880 o Chrysopa cosmeta Navas, 1904 está citada anteriormente en la provincia por NAVAS, 1908, ANDREU, 1909, NAVAS 1915 a, NAVAS, 1924, VIDAL Y LOPEZ, 1943 y HÖLZEL y OHM, 1972.

Barranco de Guadalest 27-VI-1982 2 ○ $\circ, 2$ \% 2 , 18-VII-1982 2 $\uparrow$, Benidorm 17-VII-1982 1 \%, Cocentaina 18-VII-1982 1 o , 2 , Crevillente 27-VI-1982 1 ơ, 4-VIII-1982 1 ○', Margarida 5-VIII-1982 1 \&, Montesol 28-VI-1982 2 \%, Pego 27-VI-1982 1 o*, 1 \%, 17-VII-1982 2 or ơ, 5-VIII-1982 1 \%, Pilar de la Horadada 11-VII-1982 3 \&, Puerto de la Carrasqueta 18-VII-1982 1 \&, Tárbena 27-VI-1982 1 \&, Torrellano 4-VIII-1982 1 q, Venta de Pedreguer 17-VII-1982 3 ơ ơ, 1 \%

Puede apreciarse que se trata de una especie abundante y ampliamente repartida. Su poca especificidad hace posible haberla encontrado en una gran variedad de sustratos vegetales: Ceratonia siliqua (12 ej.), Quercus ilex (8), Quercus coccifera (7), Pistacia lenticus (2), Olea europea (1). 
Anisochrysa granadensis (Pictet, 1865)

Especie atlantomediterránea, citada anteriormente en la provincia por NAVAS, 1915 a, VIDAL Y LOPEZ, 1943 y HÖLZEL y OHM, 1972.

Barranco de Guadalest 4-VIII-1982 3 or $\circ^{\prime}, 2$ 우, Cocentaina 27VI-1982 1 \&, Parcent 27-VI-1982 1 ㅇ, 5-VIII-1982 1 ○, Tárbena 27-VI-

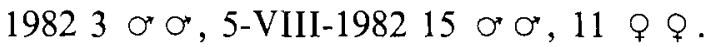

Esta especie de biología prácticamente desconocida, parece asociada a vegetación mediterránea, habiéndose colectado $30 \mathrm{ej}$. sobre Quercus ilex, 6 ej. sobre Ceratonia siliqua y 1 ej. sobre Pinus halepensis.

Anisochrysa picteti (McLachlan, 1880)

Especie muy abundante en la provincia estudiada, pudiéndose recabar citas anteriores en HÖLZEL y OHM, 1972 y en ASPÖCK, ASPÖCK y HÖLZEL, 1980.

Algorfa 4-VIII-1982 2 or ơ, Barranco de Guadalest 27-VI-1982 4 ○ ${ }^{*}$, Cocentaina 27-VI-1982 1 on, 18-VII-1982 3 ๆ $९$, Lomas de Gigi 17-VII-1982 1 ๙, 1 q, Margarida 27-VI-1982 5 q , 5-VII-1982 $1 \sigma^{\circ}, 2$ ○ ㅇ, Parcent 27-VI-1982 2 ơ ơ, 17-VII-1982 1 , Pego 17-VII-1982 1 ơ, 1 q, Pilar de la Horadada 11-VII-1982 1 ơ, 3 $\subsetneq$, Puerto de Biar 5-VIII-1982 1 \&, Puerto de Confrides 27-VI-1982 1 \%, 4-VIII-1982 $1{ }^{\circ}$, Puerto de la Carrasqueta 4-VIII-1982 1 ơ, 1 \&, Venta de Pedreguer 17VII-1982 2 ९ ․

Esta especie posee una distribución atlantomediteránea y parece confirmarse su escasa especificidad ya que ha sido colectada sobre muy diferentes fanerófitos: Quercus ilex (14 ej.), Ceratonia siliqua (11), Pinus halepensis (6), Juniperus oxycedrus (2), Quercus coccifera (2), Pistacia lentiscus (1).

Anisochrysa iberica (Navas, 1903)

Especie repartida por el Mediterráneo occidental y de la que existen numerosas referencias para esta provincia: NAVAS, 1908, ANDREU, 1909, NAVAS, 1915 a, 1924, VIDAL Y LOPEZ, 1943, HÖLZEL, 1965, HÖLZEL y OHM, 1972, y ASPÖCK, ASPÖCK y HÖLZEL, 1980. 
Margarida 5-VIII-1982 1 o*, Puerto de Biar 18-V-1982 2 ơ ơ, 2 १ ९, Puerto de Confrides 27-VI-1982 1 ơ, 1 \%, 4-VIII-1982 1 or, 1 , Puerto de la Carrasqueta 18-VII-1982 1 ơ, 1 \%

Parece preferir zonas más montanas y ha sido colectada sobre Pinus halepensis en ocho ocasiones, sobre Rosmarinus officinalis en dos $\mathrm{y}$ sobre Juniperus oxycedrus en una.

Cunctochrysa baetica (Hölzel, 1972)

Especie holomediterránea de la cual únicamente se ha colectado una hembra en Tárbena 27-VI-1982 sobre Quercus ilex.

Está citada por HÖLZEL, 1972 b y HÖLZEL y OHM, 1972 en Benisa y probablemente las citas de Chrysopa tenella Schneider, 1851 dadas por NAVAS, 1908, ANDREU, 1909, NAVAS, 1915, y VIDAL Y LOPEZ, 1943 deban referirse a la especie que estudiamos.

CONIOPTER YGIDAE Burmeister, 1839

* Aleuropteryx loewii Klapalek, 1894

Especie ampliamente distribuída por Europa y Anatolia que parece ser muy abundante en la provincia estudiada.

Algorfa 11-VII-1982 2 ơ ơ $^{\circ}$ ○ , 4-VIII-1982 1 o*, Barranco de Guadalest 4-VIII-1982 1 ơ, 1 q, Benidorm 17-VII-1982 5 ơ ơ , 4 ๆ , Calpe 17-VII-1982 1 \&, Crevillente 18-V-1982 6 ९ , 27-VI-1982 2 ơ ơ, 2 \& 우, La Aparecida 27-VI-1982 1 \%, Lomas de Gigi 17-VII19823 o $\bullet$, 4 ㅇ , Margarida 5-VIII-1982 3 \& , Parcent 17-VII-1982 1 ᄋ, 5-VII-1982 1 \%, Pilar de la Horadada 11-VII-1982 12 o $\circ, 19$ ㅇ , 4-VIII-1982 1 ○, 2 ㅇ , Puerto de confrides 4-VIII-1982 1 \%, Torrellano 27-VI-19821 .

Esta especie está ciscunscrita en la zona estudiada al Pinus halepensis sobre el que se han colectado todos los ejemplares, salvo tres que se colectaron sobre una encina aislada ubicada en un pinar, lo cual puede considerarse meramente accidental. 
* Aleuropteryx juniperi Ohm, 1968

Elemento mediteráneo expansivo, introducido en Norteamérica.

Algorfa 11-VII-1982 1 \&, Barranco de Guadalest 18-VII-1982 3 ๆ , Pego 5-VIII-1982 1 o , Puerto de Confrides 27-VI-1982 1 , Teulada 17-VII-1982 1 ○, 2 ᄋ .

Han sido colectados 6 ej. sobre Ceratonia siliqua y 1 sobre Pistacia lentiscus, Olea europea y Juniperus oxycedrus.

Aleuropteryx iberica Monserrat, 1977

Especie atlantomediterránea citada por ASPÖCK, ASPÖCK y HÖLZEL, 1980 en Alicante y como Aleuropteryx boabdil en Jijona por RAUSCH y ASPÖCK, 1978 en su descripción.

Margarida 5-VIII-1982 1 ơ, 1 \&, Puerto de la Carrasqueta 18-VII19821 o , 4-VIII-1982 2 ơ ơ. Tres ejemplares se colectaron sobre Quercus ilex y dos sobre Pinus halepensis.

Helicoconis pseudolutea $\mathrm{Ohm}, 1965$

Especie holomediterránea citada por Ohm, 1965 en Benisa y a la que he asociado las hembras de este género no colectadas con machos de segura determinación, por tratarse de la especie más frecuente de las tres halladas en Alicante.

Margarida 27-VI-1982 4 ○ , Monóvar 28-VI-1982 1 ○", Puerto de

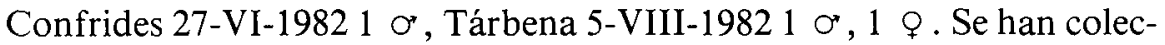
tado seis ejemplares sobre Quercus ilex y dos sobre Pinus halepensis.

* Helicoconis panticosa $\mathrm{Ohm}, 1965$

Especie probablemente holomediterránea, conocida únicamente de España y Anatolia. Se ha colectado un único ejemplar macho en Pilar de la Horadada 16-VI--1982. Como ya indiqué en MONSERRAT, $1893 \mathrm{c}$ y a la vista de esta nueva captura, me resulta sorprendente que esta especie haya sido colectada en Panticosa (Huesca) a $2.000 \mathrm{~m}$. y de nuevo aparezca una captura a $0 \mathrm{~m}$. en un ambiente totalmente xérico y completamente 
diferente al referido por OHM, 1965; el ejemplar citado se colectó sobre Pinus halepensis.

* Helicoconis hispanica Ohm, 1965

Especie totalmente desconocida, habiéndose citado del sur de España y de Marruecos, probablemente se trate de un elemento mauritano o quizás atlantomediterráneo. Con certeza puede identificarse con esta especie un macho colectado sobre Pinus halepensis el 18-VII-1982 en el Puerto de la Carrasqueta y probablemente puedan pertenecer a esta especie cinco hembras colectadas sobre Quercus ilex en la misma localidad y fecha y dos hembras más colectadas en esta localidad el 27-VI-1982 también sobre Quercus ilex.

* Coniopteryx (Coniopteryx) parthenia (Navas y Marcet, 1910)

Especie de dispersión paleártica y muy ampliamente repartida en la provincia estudiada.

Algorfa 16-VI-1982 1 , 11-VII-1982 3 ơ ơ, 5 ๆ , Benidorm 17VII-1982 3 ơ ơ, 4 \& $९$, Calpe 17-VII-1982 1 \%, Chinorlet 28-VI-1982 1 o*, La Aparecida 18-V-1982 2 ơ ơ, 3 \% $९$, Margarida 5-VIII-1982 1 \%, Monóvar 28-VI-1982 1 ○", 1 \%, Parcent 17-VII-1982 1 o", 5-VIII-1982 2 o ơ , 1 \&, Pilar de la Horadada 16-VI-1982 3 or ơ, 2 \& \&, 11-VII-1982 3 ᄋ , Puerto de Biar 18-V-1982 1 ㅇ, Puerto de Confrides 27-VI-1982 3 ơ Ơ, 18-VII-1982 1 ơ, 4-VIII-1982 3 ᄋ , Puerto de la Carrasqueta 27VI-1982 2 o $\sigma^{*}, 18-V I I-19821$ o , 1 \&, Salt 28-VI-1982 1 o*, Teulada

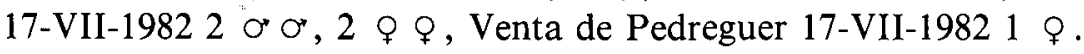

Sin duda esta especie está circunscrita en la provincia estudiada a Pinus halepensis sobre el que se han efectuado todas las capturas salvo tres ejemplares hallados sobre Juniperus oxycedrus.

* Coniopteryx (Coniopteryx) borealis Tjeder, 1930

Especie de distribución atlantomediterránea, extendida por casi toda Europa.

Algorfa 14-XI-1982 2 ơ ơ, Barranco de Guadalest 4-VIII-1982 1 o , Pego 27-VI-1982 1 ๙ , Puerto de la Carrasqueta 27-VI-1982 1 \%, 18- 
VII-1982 1 o , 1 ㅇ, 4-VIII-1982 1 \%. Tres ejemplares fueron colectados en Quercus ilex, dos sobre Ceratonia siliqua y dos sobre Populus alba.

* Coniopteryx (Holoconiopteryx) haematica McLachlan, 1868

Especie holomediterránea relativamente abundante en la provincia.

Barranco de Guadalest 18-VII-1982 1 O*, 1 \%, 4-VIII-1982 1 ơ, Cocentaina 18-VII-1982 1 ơ, 1 \&, 4-VIII-1982 1 o", 2 우, Montesol 28-VI-1982 1 ơ, 1 \&, Parcent 27-VI-1982 2 \& , Pego 27-VI-1982 1 o",

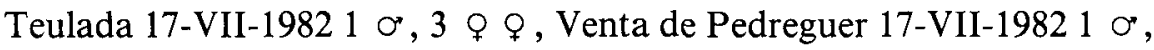
1 \%.

De estos ejemplares, 12 se colectaron sobre Ceratonia siliqua y 7 sobre Quercus ilex.

* Coniopteryx (Holoconiopteryx) drammonti Rousset, 1964

Especie holomediterránea colectada sobre Quercus ilex en una única localidad: Tárbena 27-VI-1982 2 ơ O’, 5-VIII-1982 1 ơ, 4 ๆ .

* Coniopteryx (Xeroconiopteryx) loipetsederi Aspöck, 1963

Especie probablemente holomediterránea colectada sobre Pinus halepensis en Pilar de la Horadada 4-VIII-1982 1 ơ, 1 q.

* Coniopteryx (Xeroconiopteryx) atlasensis Meinander, 1963

Nuevos datos de esta especie norteafricana siguen apareciendo, extendiendo su área de distribución a las zonas secas que limitan meridionalmente la región paleártica occidental, desde Canarias y Marruecos hasta Irán y Afganistán.

Algorfa 4-VIII-1982 1 옹, Barranco de Guadalest 18-VII-1982 2 O' $O^{\circ}, 1$ \& , Cocentaina 4-VIII-1982 1 ㅇ, Crevillente 27-VI-1982 2 우, 17-VII-1982 1 \&, Pilar de la Horadada 16-VI-1982 $1 \circ^{\circ}, 2$ \& , 11-VII19821 , Puerto de Biar 5-VIII-1982 1 \% , Puerto de Confrides 4-VIII19821 \&, Sax 28-VI-1982 1 o , Torrellano 27-VI-1982 1 ㅇ, 17-VII-1982 $1 \circ^{\circ}, 1$ ९, 4-VIII-1982 1 \%, Venta de Pedreguer 17-VII-1982 1 \% . 
Ha sido colectada sobre Ceratonia siliqua en 10 ocasiones, sobre Pinus halepensis en 5, sobre Quercus ilex en 3 y sobre $Q$. coccifera en 2.

* Coniopteryx (Metaconiopteryx) lentiae Aspöck y Aspöck, 1964

Un macho colectado en Cocentaina 18-VII-1982 sobre Querus ilex es el único dato obtenido de esta holomediterránea especie.

* Semidalis aleyrodiformis (Stephens, 1836)

Especie euroasiática muy frecuente en la provincia estudiada.

Ameradores 27-VI-1982 1 Q, Barranco de Guadalest 27-VI-1982 1

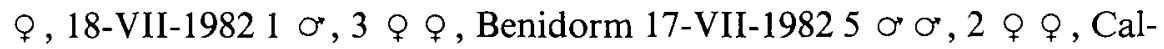

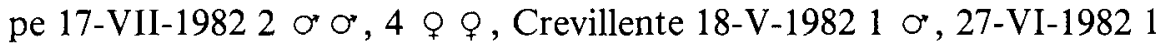
○ , 2 우, La Aparecida 18-V-1982 4 \& \& , 27-VI-1982 4 ㅇ \&, Margarida 27-VI-1982 1 \&, 5-VIII-1982 2 \&, Parcent 27-VI-1982 1 १, 17VII-1982 5 ○ ○’, 7 ㅇ , 5-VIII-1982 2 ơ ơ, 4 ๆ ㅇ, Pego 27-VI-1982 2

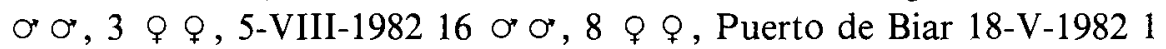
o", Puerto de la Carrasqueta 27-VI-1982 1 ơ, Tárbena 27-VI-1982 1 ९, 17-VII-1982 1 ○, 2 \% , Torrellano 17-VII-1982 2 \%, Venta de Pedreguer 17-VII-1982 9. O' $\bigcirc^{\prime}, 4$ ㅇ.

Se trata de una especie muy poco estenoica, capaz de habitar sobre muy diferentes especies vegetales, así se han efectuado 44 capturas sobre Ceratonia siliqua, 41 sobre Pinus halepensis, 8 sobre Quercus ilex, 7 sobre Olea europaea, 2 sobre Quercus coccifera y 1 sobre Pistacia lentiscus.

* Semidalis vicina (Hagen, 1861)

Especie atlantomediterránea y probablemente introducida en Norteamérica.

Tárbena 5-VIII-1982 1 ơ, 1 q, Venta de Pedreguer 17-VII-1982 2

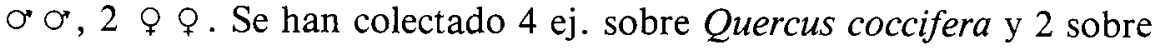
Quercus ilex. 
Especie poco frecuente y distribuída por el Mediterráneo occidental.

Algorfa 16-VI-1982 1 ๙ , Puerto de Confrides 18-VII-1982 1 ๙ , 2 우, 4-VIII-1982 4 or ơ, 5 ㅇ. . De estos ejemplares 12 se colectaron sobre Juniperus oxycedrus y 1 sobre Pinus halepensis.

* Conwentzia psociformis (Curtis, 1834)

Especie de distribución holártica de la que me ha llamdo la atención su relativa frecuencia en esta provincia, poblando medios húmedos y con densa vegetación.

Ameradores 27-VI-1982 5 ơ ơ, 1 \%, Barranco de Guadalest 18VII-1982 1 ơ, Parcent 17-VII-1982 2 ơ ơ, 5-VIII-1982 1 ơ, 1 \&, Pego 27-VI-1982 9 ơ ơ, 7 \& $q$, Torrellano 27-VI-1982 1 ơ, Venta de Pedreguer 17-VII-1982 5 ○゙ ○゙, 3 \& $ᄋ$.

Todos estos ejemplares han sido colectados sobre Ceratonia siliqua y larvas pertenecientes a esta especie ha sido colectadas sobre esta leguminosa en Ameradores y Pego, en las fechas citadas.

\section{ESPECIES CITADAS DE ALICANTE QUE NO HAN SIDO COLECTADAS:}

Como complemento al conocimiento de la fauna neuropterológica de la provincia de Alicante, se indican aquellas especies que citadas anteriormente en la provinvia, no han sido colectadas durante los muestreos efectuados. Debo indicar que me limito a exponer las referencias existentes en la bibliografía, sin descartar mis dudas respecto a la correcta determinación de algunas especies.

\section{NEMOPTERIDAE}

Josandreva sazi Navas, 1906

Desde que NAVAS, 1906 describe de Orihuela esta especie, prácticamente los únicos datos de esta especie se circunscriben a su localidad 
tipo, pudiendo añadirse las referencias de ANDREU, 1909, NAVAS, 1910 a, 1912 a, 1920, 1924, SAZ, 1925, VIDAL Y LOPEZ, 1943, TJEDER, 1974, HÖLZEL, 1975 y ASPÖCK, ASPÖCK y HÖLZEL, 1980, estos últimos indican en su cita Alicante, sin duda también deben referirse a la localidad tipo.

La especie es endémica del S.E. español y habitante de cuevas y oquedades.

ASCALAPHIDAE

Libelloides baeticus (Rambur, 1842)

Especie endémica de la Península Ibérica, ha sido citada en Alcoy por Aistleitner, 1980.

Deleproctophylla dusmeti Navas, 1914

Citada con anterioridad erróneamente como Theleproctophylla variegata (Klug, 1834) en Cox por ANDREU, 1909 y NAVAS, 1913 b, fue citada tras la descripción por NAVAS, 1914 y VIDAL Y LOPEZ, 1943.

Conocida en España y Francia, puede considerarse un elemento atlantomediterráneo, característico de zonas secas y soleadas.

MYRMELEONIDAE

Acanthaclisis occitanica (Villers, 1789)

Especie holomediterránea extendida hasta Pakistán y Afganistán y citada por VIDAL Y LOPEZ, 1943 en Orihuela.

Acanthaclisis baetica Rambur, 1842

Especie holomediterránea, citada como frecuente en Orihuela por ANDREU, 1909 y listada por VIDAL Y LOPEZ, 1943.

Myrmecaelurus trigrammus (Pallas, 1781)

Especie holomediterránea citada en Orihuela por ANDREU, 1909 y VIDAL Y LOPEZ, 1943 y en Torrelamata por MONSERRAT, 1979. 
Myrmeleon (Myrmeleon) formicarius Linneo, 1767

Especie paleártica citada por ANDREU, 1909 y VIDAL Y LOPEZ, 1943 en Orihuela. Probablemente estas citas deban referirse a $M$. gerlindae HÖLZEL, 1974.

Myrmeleon (Morter) inconspicuus Rambur, 1842

Especie holomediterránea citada en Orihuela por ANDREU, 1909, NAVAS, 1912 b, 1913 a y VIDAL 'Y LOPEZ, 1943.

Distoleon annulatus (Klug, 1834)

Especie distribuída por las zonas secas que limitan meridionalemente la región palaértica occidental, desde Marruecos hasta Irán. Está abundantemente citada en Orihuela: NAVAS, 1907, ANDREU, 1909, NAVAS, 1913 a, 1924 y VIDAL Y LOPEZ, 1943.

Neuroleon nemausiensis (Borkhausen, 1791)

Especie holomediterránea citada en Orihuela por ANDREU, 1909, NAVAS, 1913 a y VIDAL Y LOPEZ, 1943.

Creoleon aegyptiacus (Rambur, 1842)

Citada como Creagris v-nigrum Rambur, 1842 en Orihuela por ANDREU, 1909 y VIDAL Y LOPEZ, 1943.

Megistopus flavicornis (Rossi, 1979)

Especie holomediterránea, citada en Orihuela por NAVAS, 1907, ANDREU, 1909, NAVAS 1913 a, 1924 y VIDAL Y LOPEZ, 1943.

DILARIDAE

Dilar meridionalis Hagen, 1866

Especie atlantomediterránea, citada en Alicante por ASPÖCK, ASPÖCK y HÖLZEL, 1980. 
Wesmaelius (Kimminsia) navasi (Andreu, 1911)

Especie ligada a las zonas subdesérticas que limitan meridionalmente la región paleártica occidental, desde Canarias hasta Pakistán. Fue descrita de Orihuela por ANDREU, 1911 y esta localidad tipo, ha estado frecuentemente asociada a esta especie: NAVAS, 1912 c, 1924, VIDAL Y LOPEZ, 1943 y MONSERRAT, 1983 b.

Megalomus hirtus (Linneo, 1761)

Especie europea citada en Orihuela por VIDAL Y LOPEZ, 1943 y que me plantea serias dudas sobre su correcta determinación.

Megalomus tortricoides Rambur, 1842

Especie holomediterránea, citada como Megalomus tener, que fue descrita por NAVAS, 1915 b de Orihuela, por NAVAS, 1924 y VIDAL Y LOPEZ, 1943.

Megalomus pyraloides Rambur, 1842

Conocida del Mediterráneo occidental, está citada de Orihuela por NAVAS, 1908 y ANDREU, 1911.

\section{CHR YSOPIDAE}

Italochrysa stigmatica (Rambur, 1842)

Especie distribuída por las zonas secas que limitan meridionalmente la región paleártica occidental, está citada como muy común en Orihuela por ANDREU, 1909. VIDAL Y LOPEZ, 1943, recoge esta cita.

Anisochrysa subcubitalis (Navas, 1901)

Especie atlantomediterránea con numerosas referencias de Orihuela: NAVAS, 1906, 1908, ANDREU, 1909, NAVAS 1915 a, y VIDAL Y LOPEZ, 1943. 
Especie atlantomediterránea citada de Rojales y Orihuela por ANDREU, 1909, NAVAS, 1910 b, 1915 a, 1924, VIDAL Y LOPEZ, 1943 y HÖLZEL y OHM, 1972.

Chrysopa phyllochroma Wesmael, 1841.

Especie europea, extendida hasta Mongolia y citada en Orihuela por NAVAS, 1915 a, 1924 y VIDAL Y LOPEZ, 1943.

\section{CONIOPTER YGIDAE}

Aleuropteryx remane Rausch, Aspöck y Ohm, 1978

Especie únicamente conocida del S.E. español, está citada de Alicante por sus autores en la descripcion.

Tratando de hacer un somero análisis de la composición actual de la fauna neuropterológica de la provincia de Alicante, podemos reflejarlo en el siguiente cuadro, a tenor de los datos obtenidos en los muestreos:

Tipo de distribución

N. spp. colectadas

$\%$

Cosmopolita

Holártica

3

6,38

Paleártica

5

10,63

Límite meridional paleártica

3

6,38

Europea

2

4,25

Mediterránea occidental

5

10,63

Holomediteránea

19

Atlantomediterránea

Ibéricas

8

$40,42 \quad 68,08$

17,02

1

2,12

Estas proporciones podrán variar algo cuando conozcamos algo 
más sobre la distribución geográfica de muchas especies cuyos datos actuales son escasos y dispersos; sin embargo, en cualquier caso destaca la elevada proporción de elementos mediterráneos como era de esperar, alcanzando más del $68 \%$ de sus componentes.

\section{BIBLIOGRAFIA}

AISTLEITNER, E., (1980). Die Arten des Genus Libelloides Tjeder, 1972, der Iberischen Halbinsei (Neuroptera, Planipennia, Ascalaphidae). Taxonomie, Arealkunde, Phaenologie, Habitatwahl (3. Beitrag zur Kenntnis der Entomofauna der Iberischen Halbinsel). Entomofauna 1, (14): 234-297.

ANDREU, J.A., (1909). Neurópteros de los alrededores de Orihuela. Mem. Prim. Congr. nat. esp., 1908: $159-162$.

ANDREU, J., (1911). Neurópteros de la provincia de Alicante. Una especie nueva. Bol. Soc. Arag. C.N., 10: 56-59.

ASPÖCK, H., ASPÖCK, U. y HÖLZEL, H., (1980). Die Neuropteren Europas. Goecke \& Evers. Krefeld. Vol. I 495 pp. Vol. II 355 pp.

HÖLZEL, H., (1965). Neue oder wening bekannte Chrysopiden aus der Sammlung des Naturhistorischen Museums (Chrysopidae, Planipennia). Ann. Naturhistor. Mus. Wien, 68: 453-463.

HÖLZEL, H., (1972, a). Anisochrysa (Chrysoperla) mediterranea n. sp. eine neue europäische Chrysopiden-Spezies (Planipennia, Chrysopidae). Nachrichtenblatt Bayer Ent., 21(5): $81-83$.

HÖLZEL, H., (1972, b). Eine neue Chrysopiden-Art aus Südeuropa-Anisochrysa (Cunctochrysa) baetica n. sp. (Planipennia, Chrysopidae). Entomol. Zeitschr., 82(19): 217-221.

HÖLZEL, H., (1975). Revision der Netzflügler-Unterfamilie Crocinae (Neuroptera: Nemopteridae). Entomologica Germ., 2(I): 44-97.

HÖLZEL, H., y OHM, P., (1972). Die Chrysopiden der Iberischen Halbinsel (Planipennia, Chrysopidae). Faun.-Okol. Mitt., 4: 127-145.

MONSERRAT, V.J., (1979). Sobre los Neurópteros Ibéricos (II); (Neuroptera, Planipennia). Bol. Asoc. esp. Entom., 3: 17-21.

MONSERAT, V.J., (1983, a). Pterocroce capillaris (Klug, 1836) en Europa. (Neur., Plan., Nemopteridae). Neuroptera Intern., II (3).

MONSERRAT, V.J., (1983, b). Sobre los Neurópteros de las Islas Canarias, IV: Wesmaelius (Kimminsia) navasi (Andreu, 1911). (Neur., Plan., Hemerobiidae). Bol. Asoc. esp. Entom., 6 (II). 
MONSERRAT, V.J., (1983, c). Contribución al conocimiento de los neurópteros de Murcia. (Neur., Planipennia). Anales Univ. Murcia. Ciencias.

NAVAS, L. (1906). Tres neurópteros nuevos de España. Bol. Soc. Arag. C.N., 5: 134-136.

NAVAS, L., (1907). Neurópteros de España y Portugal. Broteria VI: 88-100.

NAVAS, L., (1908). Neurópteros de españa y Portugal. Broteria VII: 5-39.

NAVAS, L., (1910, a). Monografía de los Nemoptéridos (Insectos Neurópteros). Mem. R. Acad. Cienc. Art. Barcelona., VIII, 18: 341-408.

NAVAS, L., (1910, b). Crisópidos (Ins. Neur.) nuevos. Broteria IX: 38-59.

NAVAS, L., $(1912$, a). Neuroptera Fam. Nemopteridae. Genera Insectorum, 136 fasc., 23 pp.

NAVAS, L. (1912, b). Notas sobre Mirmeleónidos (Ins. Neur.). Broteria X: 29-75.

NAVAS, L., $(1912$, c). Insectes Néuroptères de Grimée. Anń. Mus. Zool. Acad. Imp. Scienc. St. Pétersbourg. XVI (1911): 528-534.

NAVAS, L., (1913, a). Mirmeleónidos (Ins. Neur.) de la fauna ibérica. Congr. Asoc. Esp. progr. Cienc, Madrid: 5-37.

NAVAS, L., (1913, b). Sinopsis de los Ascaláfidos (Ins. Neur.). Arx. Inst. Cienc. estud. Cat., 1(3): $45-142$.

NAVAS, L., (1914). Ascaláfido nuevo de España. Broteria XII: 57-60.

NAVAS, L., (1915, a). Crisòpids d'Europa (Ins. Neur.). Arx. Inst. Cienc. estud. Cat., 3(2): 1-99.

NAVAS, L., (1915, b). Neurópteros nuevos o poco conocidos (quinta serie). Mem. R. Acad. Cienc. Art. Barcelona XI, 27: 455-480.

NAVAS, L., (1920). Les Némoptérides (Ins. Néuroptères) du Muséum National de Paris. Bull. Mus. Hist. Nat. Paris, 32: 138-139.

NAVAS, L., (1924). Sinopsis de los Neurópteros (Ins.) de la península ibérica. Mem. Soc. Ibér. C.N., 4: 5-149.

OHM, P., (1965). Beiträge zur Kenntnis der Gattung Helicoconis Enderlein 1905 (Neuroptera, Coniopterygidae) nebst Diagnose zweir neuer Arten aus dem schweizerischen Nationalpark. Ergeb. wissens. Untersuch. schweizer. Nationalpark X, 53: 171-207.

PRINCIPI, M.M., (1946). Contributi allo studio dei «Neurotteri» italiani, IV Nothochrysa italica Rossi. Boll. Ist. Entom. Univ. Bologna XV: 85-102.

RAUSCH, H. y ASPÖCK, H., (1978). Zwei neue Spezies des genus Aleuropteryx Löw aus dem westlichen Mittelmeergebiet (Neuroptera, Coniopterygidae). Nachricht. Bayer. Entomol., 27(1): 9-13.

RAUSCH, H., ASPÖCK, H. y OHM, P., (1978). Zwei weitere neue Arten des Genus Aleuropteryx aus der Westpaläarktis (Neuroptera, Coniopterygidae). Entomol. Zeits., 88(5): 45-49. 
SAZ, E., (1925). La Joșandreva sazi Nav., Iberica 1925: 67-70.

TJEDER, B., (1974). Taxonomic Notes on Species of the Crocini (Neuroptera, Nemopteridae). Ent. scand., 5: 300-304.

VIDAL Y LOPEZ, M., (1943). Catálogo provisional de neurópteros de la región levantina. Graellsia 1(6): 13-24. 